\title{
Fomento de la Salud Física en Pacientes de la Facultad de Odontología de la Universidad de Cartagena: Sistematización de Experiencias
}

\section{Physical Health Promotion in Patients from the School of Dentistry at the University of Cartagena: Systematization of Experiences}

\author{
Shyrley Díaz Cárdenas ${ }^{1}$
}

Universidad de Cartagena

\section{RESUMEN}

En los últimos años, los servicios de salud han enfatizado en la atención holística y humanizada del paciente. Como parte de esos servicios, la atención odontológica no escapa a este modelo, que tiene en cuenta a la familia como entorno influyente en el individuo y a los estilos de vida como factores de riesgos o protectores de la salud; todo ello con el fin de mejorar la calidad de vida de las personas. El objetivo es realizar un resumen de la atención odontológica con enfoque en salud familiar, que se ofrece en la Facultad de Odontología de la Universidad de Cartagena, y precisar la forma como esta puede influir en los cambios de estilos de vida y, más específicamente, en el fomento de la salud física de sus pacientes. Los pacientes que acuden a la consulta odontológica de la Facultad de Odontología de la Universidad de Cartagena reciben un tipo de intervención en el que se detectan los factores de riesgos y se trabaja en la modificación de estos, y de manera particular en la disminución de enfermedades cardiovasculares y mentales.

Palabras Clave: atención odontológica, promoción de la salud, control de riesgo (Fuente: DeCS).

\section{ABSTRACT}

In recent years we have worked on holistic care and humanized patient health services. Dental care is no exception to this model of care, taking into account the family as an influence on the individual environment and lifestyles as risky or protective of health factors; all this in order to improve the quality of people's lives. The aim is make an overview of dental care with focus on family health is offered in the Faculty of Dentistry of the University of Cartagena, and as this can influence changes in lifestyles and more specifically on the promotion of the physical health of their patients. Patients who attend dental consultation of the Faculty of Dentistry of the University of Cartagena, receive a consultation where risk factors are detected and where work on the modification of these factors can begin in order to contribute to the decrease of mental and cardiovascular diseases.

Keywords: dental care, health promotion, risk management (Source: MeSH, NLM).

Odontóloga. Magíster en Salud Pública. Especialista en Salud Familiar y Gerencia en Salud. Correo electrónico:shyrley77@hotmail.com. 


\section{Introducción}

\section{Odontología con enfoque de riesgo}

En la Facultad de Odontología de la Universidad de Cartagena, desde hace aproximadamente 8 años, se inició la Atención Odontológica con Enfoque de Riesgo en Salud Familiar (1). En este sentido, sin importar el motivo de consulta, al paciente que acude a la consulta odontológica, se le realiza valoración e historias clínicas de semiología, periodoncia, endodoncia y rehabilitación oral, como también una historia clínica de salud familiar. El paciente permanece cuatro meses en la atención y su tratamiento se fracciona en cuatro cortes durante todo el periodo.

De manera más concreta, en lo que concierne a la Atención Odontológica con Enfoque de Riesgo a nivel de salud familiar, el objetivo radica en identificar los factores de riesgo de índole personal, familiar, social, laboral y comunitario que presenta el paciente, a fin de propiciar su posible modificación o eliminación, en favor del establecimiento de estilos de vida saludables (2). Esta identificación se realiza, de manera simultánea, a todos los procedimientos y tratamientos odontológicos. Así, en un primer corte, se realiza la historia clínica de salud familiar; en un segundo corte, se elaboran guías anticipatorias; en un tercer corte, se hace un seguimiento a los compromisos adquiridos en la guía anticipatoria; y en un cuarto y último corte, se elabora un informe final.

\section{Historia clínica de salud familiar}

En este orden de ideas, la historia clínica de salud familiar es un documento que contiene todos los datos del estado de salud general de la familia del paciente. Es el resultado de la elaboración de los instrumentos simplificados de valoración (3) y, como tal, busca detectar factores de riesgos presentes en el paciente y en su familia para eliminarlos o modificarlos, como también factores protectores para mantenerlos y potencializarlos (4).

Contiene seis principales aspectos, a saber:

- Identificación de la familia

- Censo familiar
- Condiciones del medio ambiente familiar e identificación de factores de riesgos relacionados con el medio ambiente familiar, ambiente socioafectivo, la morbimortalidad, las condiciones de la vivienda $\mathrm{y}$, por último, problemas familiares presentados en los últimos años que, a su vez, estén generando problemas en la salud de los miembros familiares.

- Instrumentos de salud familiar: Son los siguientes: Familiograma (5), que se utiliza para detectar problemas o patrones de comportamiento repetitivos que impliquen un riesgo en las familias; Ecomapa, que evalúa los recursos extra familiares de apoyo y soporte (6) y el Apgar Familiar (7), que evalúa la percepción de los miembros de la familia sobre su funcionalidad familiar.

- Diagnóstico

- Guías anticipatorias a implementar.

Establecida la historia clínica de salud familiar, ya en un segundo corte, al conocer los factores de riesgo y protectores de la salud, se estudian y trabajan los de más relevancia, y con base en esto se diseña una guía anticipatoria.

\section{Guía anticipatoria}

El estudiante que atiende a su paciente empieza a realizar, en forma inmediata a la realización de la historia clínica, un proceso de educación sobre los factores de riesgos a modificar. Posteriormente, entra en una conciliación con su paciente para establecer unos compromisos de cambios de comportamiento, hábitos y estilos de vida que mejoren su salud. Este compromiso queda sentado en un documento llamado Guía Anticipatoria.

En el marco de la guía anticipatoria, también cumplen un papel fundamental las guías preventivas, que son una condensación de las diferentes intervenciones en promoción de la salud y prevención de la enfermedad que se efectúan para intervenir a las poblaciones. Estas contienen estrategias de consejería, filtrado y medidas específicas de protección (8).

En un contexto donde la prevención en los sistemas de salud es cada vez más importante, las guías 
preventivas constituyen la condensación de un esfuerzo por ofrecer al personal de salud las medidas e intervenciones que según la evidencia científica tienen eficacia y efectividad para la prevención primaria, a saber: promoción general de la salud o prevención de enfermedades específicas antes que aparezcan; y prevención secundaria: descubrimiento precoz de enfermedades o prevención de sus posibles complicaciones.

Por lo general, las guías preventivas incluyen tres tipos de intervenciones:

- Consejería. Son un conjunto de recomendaciones generales o específicas para evitar riesgos a la salud y mantener una salud integral. Se pueden incluir, por ejemplo, la recomendación sobre lactancia materna exclusiva, el almacenaje adecuado de medicamentos y venenos, la recreación regular en familia, cesación del tabaquismo, etc.

- Medidas específicas de protección. Son intervenciones dirigidas a evitar riesgos específicos para la salud o enfermedades específicas. Entre ellas se encuentran: la vacunación, la administración de hierro y calcio durante el embarazo, el uso de casco al conducir moto o bicicleta (aunque esto último también se puede presentar como consejería), etc.

- Tamización o pruebas de filtro. Integradas por medidas para la demostración temprana de algún riesgo o enfermedad. Puede ser, a su vez, de tres tipos:

Anamnesis. Es en sí la aplicación de preguntas o cuestionarios estructurados, tales como cuestionarios de depresión, cuestionario para descubrir alcoholismo, etc.

Técnicas de examen físico. La toma de presión arterial, la determinación del índice de masa corporal, el examen clínico de senos, testículos, etc., hacen parte de este tipo de técnicas.

Pruebas de laboratorio. Por ejemplo, la citología cérvico-vaginal para descubrir temprano el cáncer de cérvix, el perfil lipídico en adultos para el hallazgo de dislipidemias por su asociación con enfermedad cardiocerebrovascular.

En un tercer corte, se hace el seguimiento de los compromisos adquiridos por el paciente semanalmente, y en un cuarto corte, un detallado informe final de todo lo realizado con el paciente a nivel de salud familiar.

\section{Cambios en los estilos de vida de los pacientes desde la consulta odontológica}

Son muchas las experiencias gratificantes obtenidas en cuanto a cambios de estilos de vida a partir de la atención en salud bucal de los pacientes. Una de estas experiencias corresponde a una paciente de 34 años de edad que acudió a la consulta odontológica para la realización de una prótesis fija de tres unidades en el sector posterior superior de la boca. La paciente presentaba un nivel de escolaridad secundaria, era soltera y trabajaba como mensajera en una empresa privada.

Al ser examinados los factores de riesgo relacionados con su estilo de vida después de la realización de la historia clínica, se encontraron:

- Antecedentes de diabetes en la familia paterna, y de hipertensión arterial en la familia materna (9).

- $\quad$ Trabajo extenuante y estresante, en horario de 7 a.m. a 6 p.m., tiempo en el cual debía llevar toda la mensajería asignada, agravado por el hecho de que la paciente no contaba con un transporte particular propio y debía movilizarse en servicio urbano.

- Poco consumo de agua, frutas y vegetales en su dieta habitual.

- Un sobrepeso leve, pues, al realizarse su Índice de Masa Corporal (IMC),estaba por encima de 29 $(10,11)$

- Ausencia de actividad física como prácticas de ejercicios diarios o de un deporte (12).

Al ser analizados estos factores, se decidió realizar educación durante dos semanas continuas mientras asistía a la consulta odontológica, sobre todo lo relacionado con un estilo de vida saludable, que se opone al sedentarismo y, en cambio, resalta los beneficios y consecuencias del ejercicio físico. Con posterioridad a la educación realizada, a la paciente se le propusieron las siguientes recomendaciones en su guía anticipatoria:

- Caminar mínimo durante 30 minutos 3 días a la semana, de 5:30 a. m. a 6 a.m. 
- Tomar 8 vasos de agua al día.

- Realizar controles anuales médicos para revisar su perfil lipídico, sus niveles de glicemia y su presión arterial, además de todos los chequeos necesarios para la edad de la paciente y su sexo.

- Comer diariamente, a las 9 a. m. y a las 4 p. m., una fruta al natural.

- Acompañar el almuerzo rutinario con una ensalada natural de vegetales verdes oscuros.

Una vez realizadas estas recomendaciones en la guía anticipatoria, la paciente insistía en que ella no necesitaba hacer ejercicio, debido a que por su oficio de mensajería se mantenía activa.

Si bien ello tiene mucho de cierto y tal actividad la protege frente a oficios y profesiones en los que se debe permanecer mucho tiempo sentado frente a un computador, por ejemplo, el objetivo con la paciente era concientizarla de la importancia de adquirir y mantener una buena salud física utilizando como estrategia el ejercicio, que necesita la unión de la mente y el cuerpo para lograr el estado deseado. Al respecto, se ha comprobado que la práctica de actividad física bien planteada está asociada a una serie de beneficios psicológicos, como la mejora del carácter y de la calidad de vida, la reducción del estrés, la adopción de un autoconcepto más positivo; y la reducción del estado de ansiedad, neurosis y depresión, sin distinción de edad ni sexo (13). A partir del planteamiento de John Locke, la relación entre salud mental y física se reitera en la bibliografía médica, pues con él se demostró que la actividad física está relacionada con: disminución de la ansiedad y depresión, aumento de la autoestima, mejoría académica, disminución del uso de alcohol, tabaco y marihuana, y la percepción de una vida más satisfactoria (13). La actividad física es decisiva en estos comportamientos, que resultan determinantes para la calidad de vida.

Para lograr la motivación del paciente y el cumplimiento de este importante compromiso, el estudiante que atendió a la paciente se ideó un cuadro de control de registro diario de las actividades de salud física a realizar, junto con el registro del tiempo empleado y el tipo de ejercicio realizado.

En la primera semana de seguimiento, la paciente comenzó a caminar tres días durante 30 minutos. En la segunda semana lo asumió cuatro días. Para la tercera semana lo hizo cinco días y ya para la cuarta, por iniciativa propia, decidió empezar a trotar, pues notó una disminución de peso con el pasar de los días y comentaba que esto la tenía muy motivada.

Ya hacia el final del cuarto corte, la paciente logró bajar definitivamente de peso, alcanzando un IMC de 24,5. También cambió y mejoró sustancialmente hábitos y comportamientos, por lo que se sentía más contenta y optimista personal y socialmente hablando, esto es, con un mejor y nuevo estilo de vida.

Como esta experiencia, son muchos los estilos de vida que se han ido cambiando entre los pacientes que asisten a la Facultad de Odontología de la Universidad de Cartagena, impactando positivamente en la solución de divorcios, manejo adecuado del duelo, acompañamiento y educación a las familias sobre las enfermedades del paciente, aumento del consumo de agua, manejo del estrés y la ansiedad entre otros. En todos los casos se aplican diferentes estrategias de educación e intervención, dependiendo de la dinámica y problemática familiar.

\section{Conclusiones}

A partir de vivencias como las antes referidas, se puede decir que todo paciente que acude a la consulta odontológica de la Facultad de Odontología de la Universidad de Cartagena, además de recibir una atención y una solución a sus problemas de salud bucal, recibe una intervención en salud familiar, mediante la cual se detectan sus factores de riesgo y se trabaja en la modificación de los mismos. A todos los pacientes se les evalúan en específico los factores de riesgo relacionados con su salud física, se les educa en estilos de vida saludables y en fomento del ejercicio, como también se les practica un seguimiento y un control de sus actividades físicas. Con todo esto, se propician cambios saludables en sus comportamientos y el mantenimiento de los mismos, de modo que se favorezca su salud mental y física y mejore su calidad de vida. De esta manera, se contribuye concretamente a la disminución de factores de riesgos relacionados con enfermedades de origen cardiovascular y mental en la ciudad de Cartagena, y estas intervenciones y actividades se ven reflejadas de manera positiva en los indicadores nacionales de morbilidad y mortalidad. 


\section{Referencias}

1. Louro I. Modelo de salud del grupo familiar. Rev Cubana de Salud Publica 2005; 31(4).

2. Rodríguez CA, Valiente ZC, Tan N. Las familias saludables y los factores determinantes del estado de salud. Rev Cubana Estomatol. 2001; 38(3):165-169.

3. Jáuregui RC, Suarez CP. Promoción de la Salud y Prevención de la Enfermedad: Enfoque en salud Familiar. 2nd ed. Panamericana; 2004.

4. Amaya P. Instrumento de Riesgo Familiar Total. Manual. Aspectos teóricos, psicométricos de estandarización y de aplicación. RFT: 5-33 (Tercer tomo). Santa Fe de Bogotá: Editorial Universidad Nacional de Colombia; 2004.

5. El Familiograma medicina familiar en la práctica: Nivel familiar de la Práctica. Ministerio de Salud. Cali: Catorce Editores; 1997.

6. Arias L, Alarcón M. El Ecomapa. Medicina Familiar en la Práctica: Nivel familiar de la Práctica. Ministerio de Salud. Cali: Catorce Editores; 1997.

7. Smilkstein G. The Family APGAR: A proposal for a family function test and its use by physicians. J Fam Prac. 1979; 6: 1231-1239.

8. Pineda C. Guías preventivas por ciclo vital individual en Colombia. Colomb Med. 2006; 37 (2): 65-74.

9. Espinoza GF, Ceja EG, Trujillo HB, Uribe AT, Abarca HP, Flores VD. Análisis de los factores de riesgo de la hipertensión arterial en Colima, México. Rev Panam Salud Pública. 2004; 16(6):402-407.

10. Kronenberg HM, Melmed S, Polonsky KS, Larsen PR. Williams Textbook of Endocrinology. 11th ed. Philadelphia: Saunders Elsevier; 2008.

11. Skelton J. Overweight and obesity. In: Kliegman RM, Jenson HP, Stanton BF, eds. Nelson Textbook of Pediatrics. 18th ed. Philadelphia, Pa: Saunders Elsevier; 2007.

12. Escobar J. Actividad física como estilo de vida saludable: Criterios básicos. Rev Med Risaralda. 2003; 9(2): 43-55.
13. Kuczmarski MF, Kuczmarski R, Najjar M. Effects of age on validity of self-reported height, weight, and body mass index: findings from the third National Health and Nutrition Examination Survey, 1988-1994. J Am Diet Assoc. 2010; 110 (1): 28-34. 